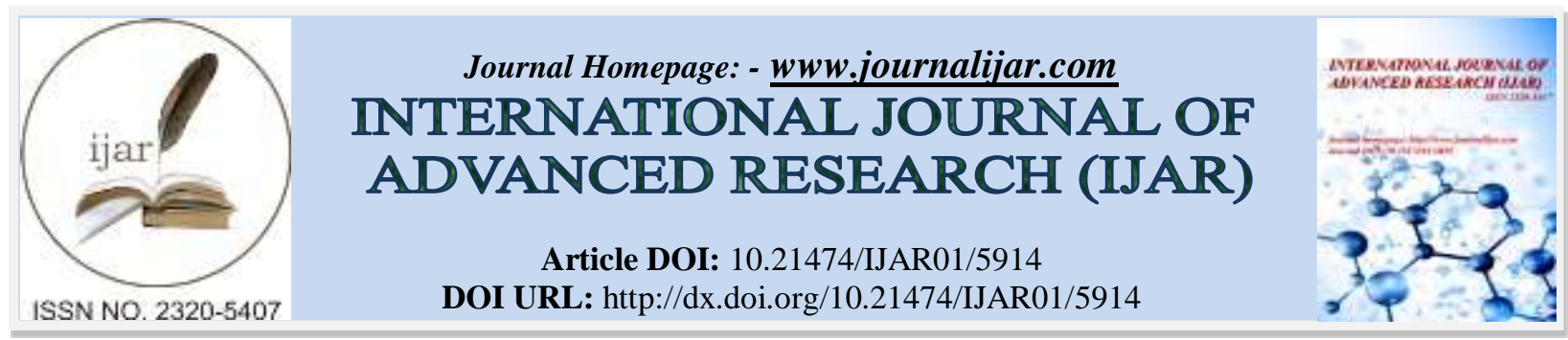

RESEARCH ARTICLE

\title{
RISK FACTORS ASSOCIATED WITH FAMILY VIOLENCE IN PRIMARY EDUCATION STUDENTS.
}

\section{María Eugenia López Caamal ${ }^{1}$, Betty Sarabia Alcocer ${ }^{2}$, María Guadalupe Jaimez Rodríguez ${ }^{1}$, Alma Delia Sánchez Ehuán ${ }^{1}$, María Concepción Ruíz de Chávez Figueroa ${ }^{1}$ and Ariosto Segura Escamilla ${ }^{1}$.}

1. Instituto Campechano -School of Social Work.

2. Faculty of Medicine of the Autonomous University of Campeche.

\section{Manuscript Info}

Manuscript History

Received: 19 September 2017

Final Accepted: 21 October 2017

Published: November 2017

Key words:-

Family violence, suicide, primary education.

\begin{abstract}
Through a correlational, cross-sectional field and mixed study, guided by a systemic approach; it was determined that family violence is a risk factor for suicide. In the study an instrument was applied in order to measure the degree of intrafamiliar violence and the risk of suicide, this in a sample of 1624 students of $5^{\circ}$ and 6th graders from 22 schools enrolled in the 2014-2015 school year. Based on the results obtained, it is found that $25 \%$ (414) of the children studied present a risk of suicide to some extent, whether low, medium or high risk; Likewise, it is found that $31 \%$ (505) of the children studied presented intrafamiliar violence.
\end{abstract}

Copy Right, IJAR, 2017,. All rights reserved.

\section{Introduction:-}

Family violence is a phenomenon that is present throughout the world and Mexico is not foreign to it, situations such as poverty, machismo, addictions, etc., are related to family violence.

The Ministry of Health in 2010 reports in national figures that children suffer family violence by $6 \%$, occupying the third place as a group, after adults with $60 \%$ and young people with $26 \%$. In the same source it is presented that Campeche occupies the second place with 249 cases in population of 0 to 9 years that could have suffered family violence, being the State of Mexico that occupies the first place with 735 cases. On the other hand, in persons aged 10 to 14 years who could have suffered intrafamiliar violence, the second place also has 254 cases, with the State of Mexico occupying the first place with 280.

Family violence has a cost in Latin America of just over 160 billion dollars, reports the Inter-American Development Bank (IDB). That problem for Mexican society will cost him at least 30 years to reverse his negative psychological effects. It affects 50 percent of the female population and is rooted in seven out of 10 households in the country ( Women's Communication and Information, 2007)

The objective of this study is to determine the incidence of family violence in primary school students in the city of San Francisco de Campeche, in the 2014-2015 school year, in addition to make proposals for prevention and attention to violence intrafamiliar in students of primary education of the city of San Francisco de Campeche.

Psychologically, violence is considered as a figure of aggressiveness, which is registered in reaction to real or alleged injustices suffered, as an intention to realize one's personality, or as an inability to pass from the principle of pleasure to the principle of reality, with the consequent intolerance to frustration. ( ${ }^{\text {Galimberti, 2002 }}$ ) 
In this definition, an element appears to appear to be the principal element in violence: aggression.

Resuming aggression as a substantial element of violence, Apodaca (1995) mentions that violence is a manifestation of power or dominion with the intention of controlling someone, being the most obvious way to exercise is through aggression, understanding to it as the purpose of physically or psychologically damaging some person.

On the other hand, the World Health Organization (1996) defines violence as: The deliberate use of physical force or power, whether threatening or effective, against oneself, another person or a group or community, which causes or is likely to cause injury, death, psychological damage, developmental disorders or deprivation $\left({ }^{\mathrm{WHO}, 2013}\right)$.

These definitions allow us to define violence as a coercion exerted on oneself or others whose main element is aggressiveness, which is probably originated in the reaction to perceived injustices are these true or fictitious, they also distinguish between interpersonal violence and suicidal behavior and armed conflict.

It also covers a wide range of acts that go beyond the physical act to include threats and intimidation. In addition to death and injury, the definitions also encompass the many consequences of violent, often less noticeable, behavior such as mental damage, deprivation and developmental deficiencies that compromise the well-being of individuals, families and communities. This is supported by Apodaca who mentions that "aggression can not only be directed to the body of a person or to material values, but to the human essence itself, morally or philosophically considered. Aggression can even be present in the very formation of consciousness "(Apodaca Rangel, 1995).

Taking up the definition of violence from the psychological point of view that has been exposed in previous lines, it is interesting to analyze the assertion that aggressiveness arises in reaction to situations experienced by an individual that are perceived as unfair, this would suppose a natural-evolutionary origin of the aggressiveness and consequently of the violence in the human being.

Interpersonal violence is divided into two subcategories:

1. Family or couple violence: In most cases, it occurs between family members or sentimental partners, and usually happens in the home, but not exclusively.

2. Community violence: occurs between unrelated individuals that may or may not be known; usually happens outside the home.

The first group includes forms of violence such as child abuse, violence against the couple and abuse of the elderly. The second group includes youth violence, random acts of violence, rape and sexual assault by strangers, and violence in establishments such as schools, workplaces, prisons and nursing homes.

When violence, in any of the types already described, is exercised against, a member of a family is spoken of domestic violence or domestic violence. This violence in a problem that is gaining strength year by year so it is urgent to study it.

Family violence is a major social problem. Their consequences put at risk the health and even the life of the population groups that are most vulnerable according to their sex, age and physical condition: women, girls, children, elderly, elderly and disabled people. This violence; in most cases, is carried out by men against women and girls. (Secretaría de Salud Guadalajara, 2002).

Angela Hernández (1997) mentions that family violence is a problem for both men and women, they exchange the roles of aggressors and aggressors, they change their position in the conflict according to their escalation.

This exposes that, despite the family violence most commonly exercised against infants and especially women, it also exists against men, which would lead to the conclusion that family violence is the product of a conglomeration of factors such as sex, the environment - especially if it is tense - values, beliefs, etc.

Sierra, Macana and Cortés (2014) mention that family violence is dynamic, fluctuating, but it responds to the conditions, tools and options for conflict resolution learned and reinforced in the context in which it interacts. So violence is a learned behavior based on family and social models that define it as a valid resource for resolving conflicts; we recognize typologies and family environments that establish within their customs, values and rules the 
establishment of a culture based on patterns of aggression that is deteriorating, affecting and strengthening dysfunctional family dynamics.

That is, family violence is the result of dysfunctional social and family dynamics, malfunctioning of a system in which members of a family are immersed, and highlights the fact that each member of a family is an active participant in this violence.

\section{Material and Methods:-}

The scope of the present investigation is of correlation, through which the intrafamiliar violence was analyzed. It is a cross-sectional, descriptive and observational study.

In the research work it was determined that the methods used to obtain the information were the questionnaire (questionnaires and interviews) and, for the presentation of results, the explanatory, through the presentation of absolute and percentage data, according to the case. The explanatory method to explain the arguments of the analysis that was made using the obtained results. The graphs and the theoretical referents; the inquiry for the investigation itself that is carried out that allows to know in more detail the problematic; and percentage in the sense of applying the statistical procedure to determine percentages that give information on the incidence of what is studied.

The population that represented the subjects of study in the investigation are the students of primary education of the city of San Francisco de Campeche that comprise a range of age of 10 to 14 years.

The population considered as a universe were primary education students enrolled in the 72 public schools of the city of San Francisco de Campeche.

The sample population was represented by 30 percent of the public elementary schools that correspond to 22 schools and students enrolled in grades 5 and 6 of the morning or afternoon shift. This percentage seeks to guarantee the reach of a minimum of $20 \%$, taking into account all possible inconveniences that could arise during the collection of information in the field.

The measuring instrument has four sections: General data, family violence, family and risk situations; consists of 15 questions with four response options each. At the end of the questions has a section for qualitative information with five questions, free answers, with no options that will allow checking the congruence with the multiple answer questions.

To facilitate its application was applied to the group, supporting the interviewer with reading the questions for the respondent to follow and mark their response. The instrument was piloted in two primary education schools, to verify the comprehension of the questions by the respondents, in order to make the necessary modifications.

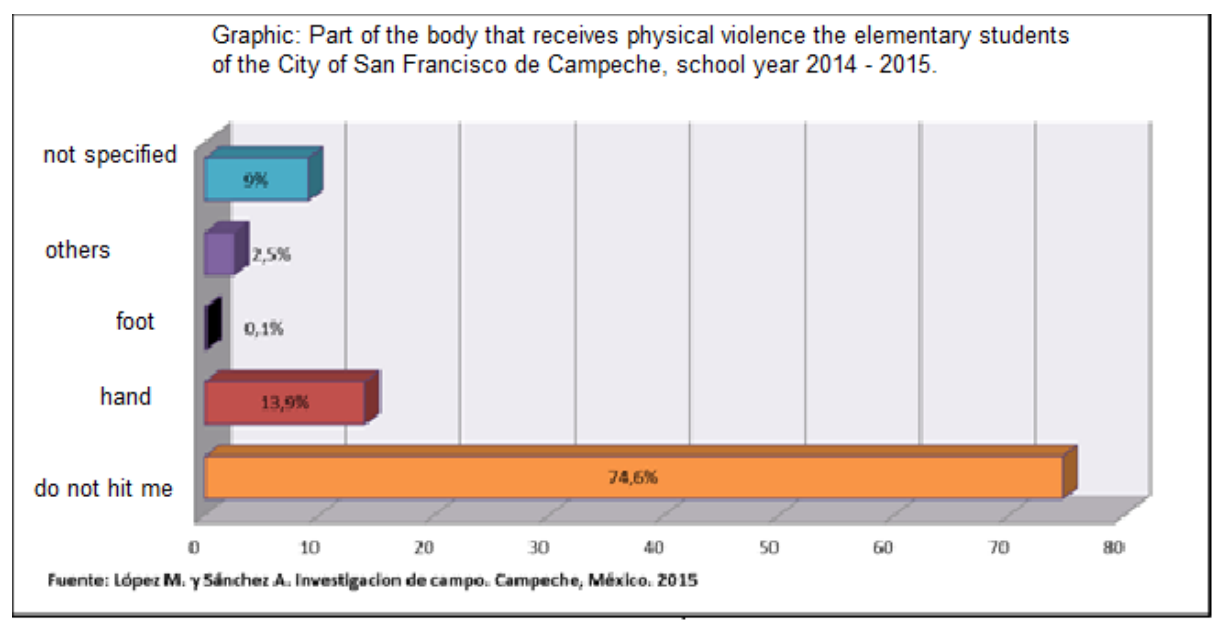

Graph 01:- The hand is the part of the body that they use most to exercise violence in 13\%. 


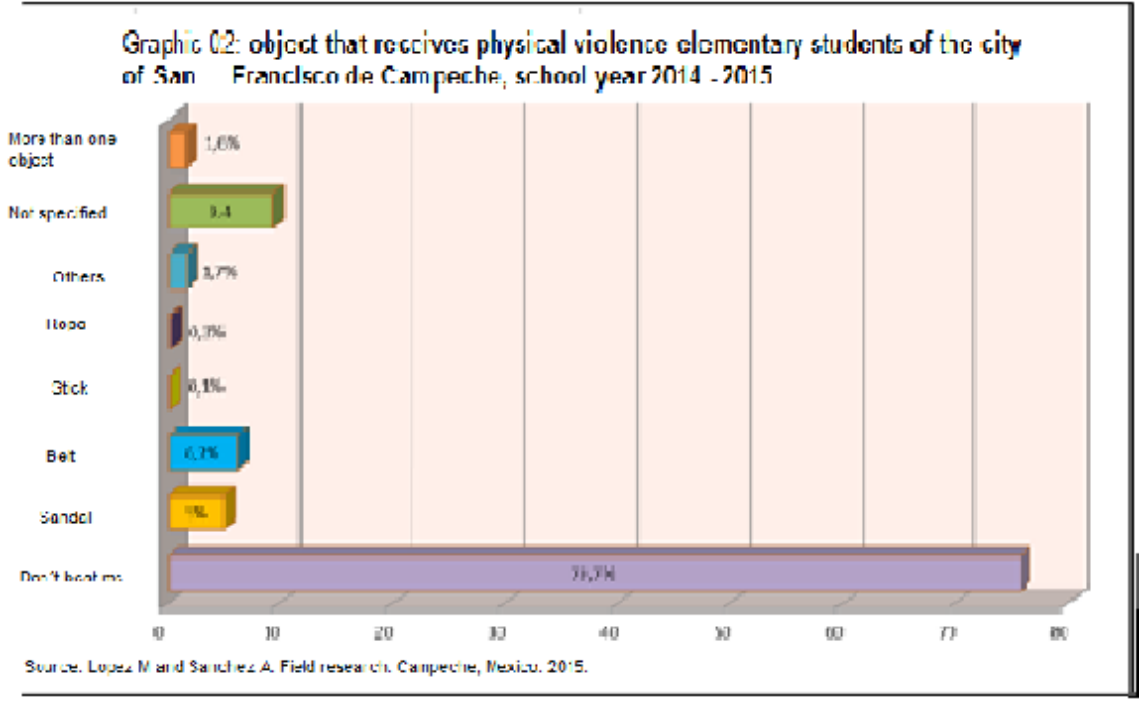

Graph 02:- The belt with $6.2 \%$ was the object with which they receive physical violence.

Graph 03: Objective and part of the body not specified with which physical violence receives the elementary students of the city of San Francisco de Campeche, school year 2014 - 2015.

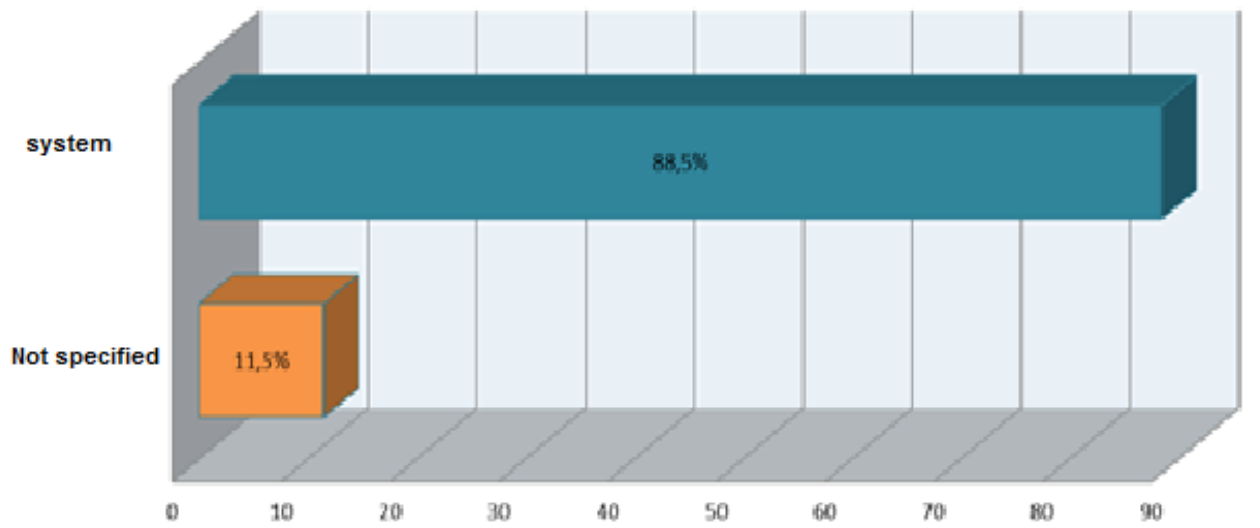

source. Lopex M and sanchez a. field research. Campech, Mexico. 2015

Graph 03:- The 11.5\% (186) correspond to the unspecified object and body part with those that exert physical violence people towards primary students.

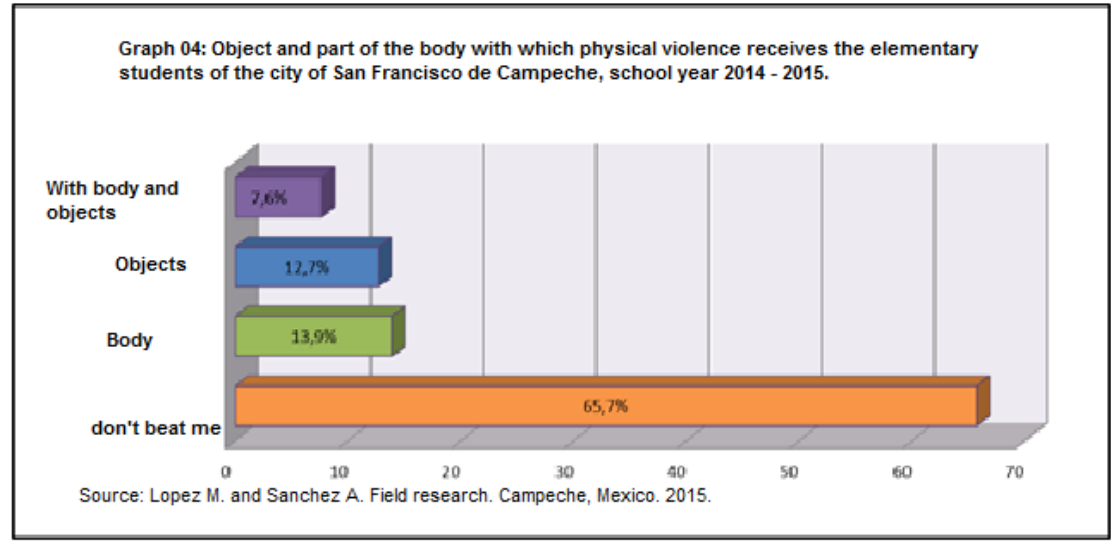

Graph 04:- It represents the percentages with respect to the objects or parts of the body with which people exert physical violence in the primary students, the results were: with the body $13.9 \%$ (226) 


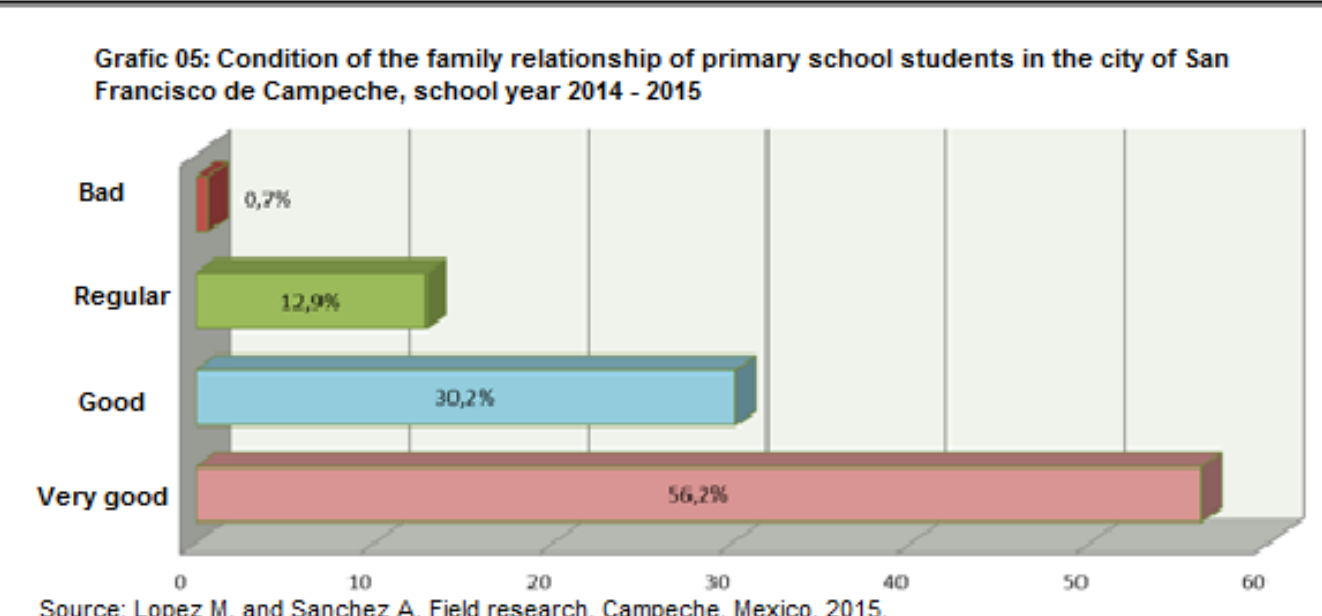

Graph vs:- 1 he condition of tamily treatment within the nome, $56.2 \%$ (Y15) answered that it is "very good"

Graph 06: method that solves the family problems the members of the families of the primary students of the city of San Francisco de Campeche, school year 2014 - 2015.

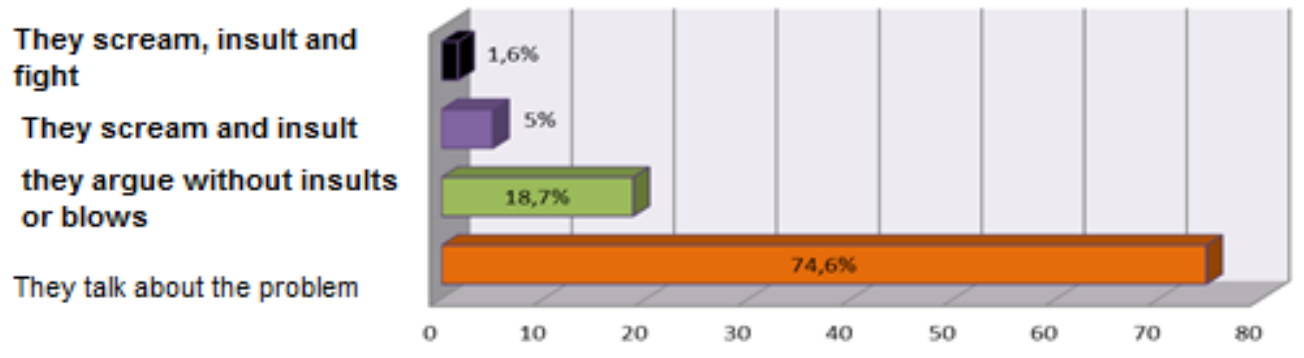

Source: Lopez M. and Sanchez A. Field research. Campeche, Mexico. 2015.

Graph 06:- Family problems solve it at home, obtaining 74.6\% (1212).

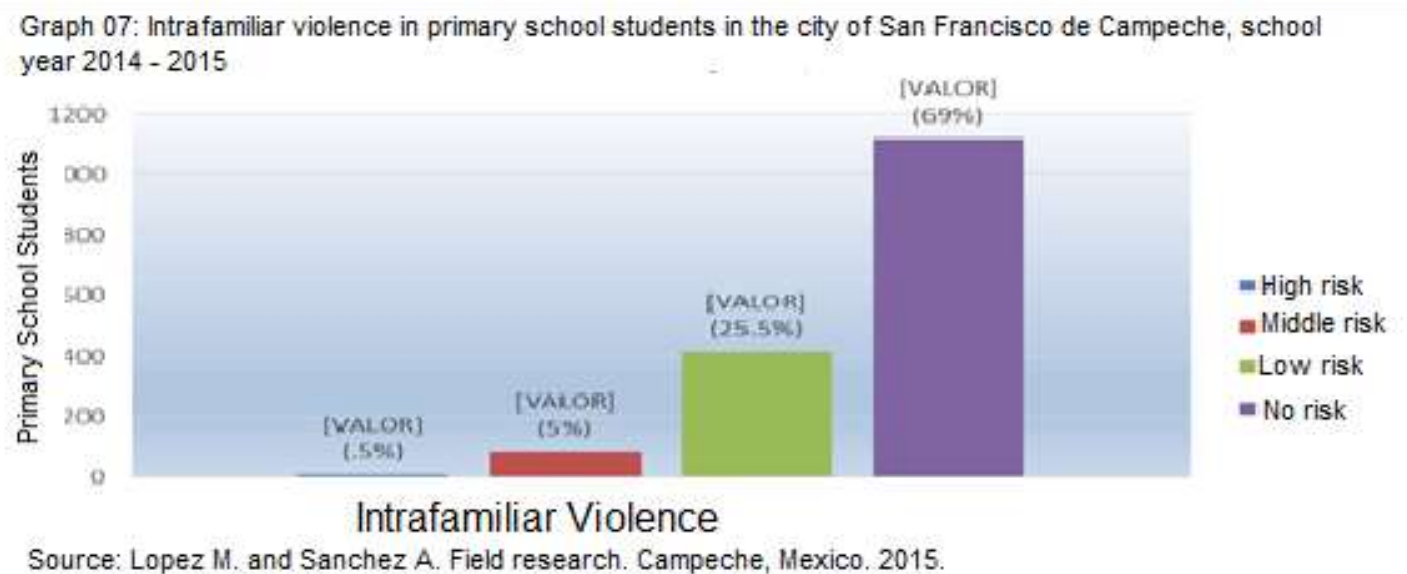

Graph 07:- It was obtained that .5\% (8) presented high risk, 5\% (84) middle risk, 25.5\% (413) low risk, and 69\% (1119) presented no risk 


\section{Results:-}

Figure 01 presents information about the body part that people use to exert physical violence on primary students, the most common being $13.9 \%$ (225), other parts of the body $2.5 \%$ (40), foot $0.1 \%$ (2) and 9\% (146) chose not to specify the part of the body with which they are hit.

Graph 02 shows the percentage of people using physical violence in primary school students, using the following: belt $6.2 \%$ (100), flip-flops $5 \%$ (81), rope $0.3 \%$ (5), stick $0.1 \%(2) ; 9.4 \%$ (152) did not specify the object with which they hit him, $1.7 \%$ (28) mentioned other objects, and 1.6\% (26) mentioned that they hit him with more than one object.

Graph 03 shows the percentage of $11.5 \%$ (186) corresponding to the unspecified object and body part with those that exert physical violence to the primary students.

Graph 04, representing the percentages with respect to the objects or parts of the body with which people exert physical violence in primary students, the results were: with the body $13.9 \%$ (226), with objects $12.7 \%$ (207), with both, that is, body and objects $7.6 \%$ (124). This means that physical violence is done directly between the person and the children, in this case in the elementary students.

Graph 05 refers to one of the questions asked regarding the family area, specifically the condition of family treatment within the home, 56.2\% (913) answered that it is "very good", 30.2\% (491) they said that the treatment inside their home eta "good; $12.9 \%$ (209) stated that it was "regular" and $0.7 \%$ (11) stated that it was "bad."

Graph 06 was found in the answers given by the students that the family problems solve it at home. Obtaining that $74.6 \%$ (1212) said they talk about the problem, $18.7 \%$ (304) mentioned that they discuss without arriving at insults or beatings, $5.0 \%$ (82) stated that they are screaming and insulting themselves and $1.7 \%$ (26) indicated that besides insults and shouting there are blows. In such a way as to relate the results of those who are shouted and insulted and those who insult themselves and beat inside the family, which translates into physical and verbal violence in $6.7 \%$, that is, in 108 families of the students of primary school of the City of San Francisco de Campeche.

Figure 07 shows the results of intrafamiliar violence in primary school students.

It was found that 5\% (8) represent high risk, 5\% (84) mean risk, 25.5\% (413) low risk, and 69\% (1119) present no risk; grouping the results of high, medium and low grade, it is obtained that $31 \%$ of the students present some degree of intrafamiliar violence, in absolute numbers, it is equivalent to 505 students in this condition. Finding the results it is found that $5.5 \%$ (92) primary students live intrafamiliar violence by being in middle and high risk.

\section{Conclusions:-}

After carrying out the corresponding quantitative and qualitative analysis of the results obtained in the investigation, a number of relevant data are obtained. Highlighting the fact that, although the percentage of children receiving treatment "regular and bad" in their homes is low (14.3\%), when reviewing the absolute data shows that the percentage represents 232 children receiving this unfavorable treatment, likewise 170 children-as (10.5\%) are violated with shouts, insults and even beatings a situation that is undoubtedly worrying.

In the specific section of physical violence, it was found that it is the mother who most exerts this type of violence on children; use the hand to perform it and the object most commonly used to carry it out is the belt.

The above, together with other data presented in the report, allow to determine the existence of intrafamily violence in $31 \%$ (505) of the families of the students who participated in the study, this allows to affirm one of the initial hypotheses of the investigation, which shows that there is intrafamily violence in at least $30 \%$ of primary school students. 